\title{
Revista Colombiana de

\section{Costo-efectividad del abordaje radial comparado con el femoral en la arteriografía coronaria diagnóstica e intervencionista}

\author{
Henry Borja Romero ${ }^{\mathrm{a}, \mathrm{b}}$, Yaneth Gil-Rojas ${ }^{\mathrm{c}, \mathrm{d}}$, Juan Manuel Senior ${ }^{\mathrm{e}, \mathrm{b}}$, \\ Camilo Castañeda-Cardona ${ }^{\mathrm{C}}$ y Diego Rosselli ${ }^{\mathrm{f}, *}$
}

\author{
a Servicio de Hemodinamia, Hospital San Vicente Fundación, Medellín, Colombia \\ b Universidad de Antioquia, Medellín, Colombia \\ c NeuroEconomix, Bogotá, Colombia \\ d Facultad de Economía y Ciencias administrativas, Pontificia Universidad Javeriana, Bogotá, Colombia \\ e Cardiología Clínica e Intervencionista, Hospital San Vicente Fundación, Medellín, Colombia \\ f Departamento de Epidemiología Clínica y Bioestadística, Facultad de Medicina, Pontificia Universidad Javeriana, Bogotá, \\ Colombia
}

Recibido el 5 de septiembre de 2017; aceptado el 22 de marzo de 2018

Disponible en Internet el 2 de julio de 2018

\author{
PALABRAS CLAVE \\ Angiografía \\ coronaria; \\ Intervención \\ coronaria \\ percutánea; \\ Arteria femoral; \\ Arteria radial; \\ Costos y análisis \\ de costo
}

\begin{abstract}
Resumen
Objetivo: Evaluar la costo-efectividad del acceso arterial radial comparado con el femoral en la realización tanto de arteriografía coronaria diagnóstica como de angioplastia coronaria transluminal percutánea.

Métodos: Se construyó un modelo de árbol de decisión para determinar costos de las dos intervenciones en pacientes adultos con sospecha de enfermedad cardiovascular. Para el análisis de efectividad se evaluó la frecuencia de complicaciones mayores y menores en ambos abordajes. La perspectiva para los costos fue la del tercero pagador (sistema de salud colombiano); se incluyeron solo costos médicos directos asociados a los procedimientos y las complicaciones, expresados en pesos colombianos de 2016 (1 USD = COP 3.051).

Resultados: El costo promedio estimado de la arteriografía coronaria con abordaje radial fue $\$ 1.384 .945$ y el femoral de $\$ 1.474 .543$. En angioplastia coronaria transluminal percutánea se estimó un costo ponderado de $\$ 8.037 .743$ en el abordaje radial y $\$ 8.319 .178$ en el femoral. El abordaje radial fue dominante para los eventos adversos cardiovasculares mayores respecto al femoral. Tanto en angiografía diagnóstica como en intervención el abordaje radial evita 0,9\% de los eventos adversos cardiovasculares mayores y 1,6\% de los sangrados mayores, pero presenta más complicaciones menores que el abordaje femoral.
\end{abstract}

\footnotetext{
* Autor para correspondencia.

Correo electrónico: diego.rosselli@gmail.com (D. Rosselli).
} 


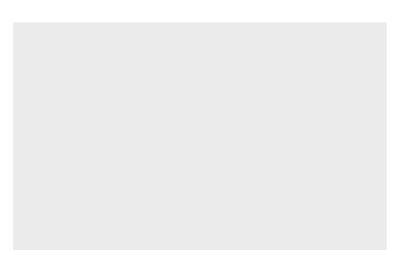

KEYWORDS
Coronary
angiography;
Percutaneous
coronary
intervention;
Femoral artery;
Radial artery;
Costs and cost
analysis

\section{Introducción}

En Colombia, a medida que ha aumentado la expectativa de vida, también lo han hecho las enfermedades degenerativas relacionadas con la aterosclerosis cardiovascular, con el consecuente aumento en los costos de atención ${ }^{1}$. Las enfermedades cardiovasculares han venido consolidándose como la principal causa de muerte de los colombianos, y la proporción de muertes por infarto de miocardio ha venido en aumento en el periodo 1960-2012 ${ }^{2}$. El hecho de que la enfermedad coronaria, por su alta morbimortalidad y alto impacto social y económico se convierta en un problema de salud pública, obliga a diseñar planes y acciones de control ${ }^{3}$. Según estadísticas del Departamento Administrativo Nacional de Estadísticas (DANE), en Colombia la tasa de mortalidad cardiovascular es de 130,2 por 100.000 habitantes, muy superior a la tasa de mortalidad de neoplasias ${ }^{4}$. De acuerdo con las proyecciones de un estudio del Foro Económico Mundial realizado junto con la escuela de Salud Pública de Harvard los costos directos e indirectos por las enfermedades cardiovasculares en el mundo pasarían de US\$863.000 millones en 2010 a US\$1,04 billones en $2030^{5}$.
En tal sentido, son varias las estrategias disponibles para combatir el incremento rápido en la mortalidad, pasando por diferentes campañas de promoción y prevención como también los tratamientos encaminados a tratarlas una vez han aparecido. Es así como el intervencionismo coronario percutáneo (ICP) se ha convertido en el método de referencia actual para visualizar el árbol coronario y las lesiones que comprometen su calibre ${ }^{6}$. Dicho procedimiento se realiza con fines diagnósticos y terapéuticos, y la decisión de llevarlo a cabo debe estar basada en el análisis cuidadoso del riesgo-beneficio del paciente. De acuerdo con el Colegio Americano de Cardiología (ACC) y la Asociación Americana del Corazón (AHA) la cateterización y la intervención coronaria se encuentran indicadas en el tratamiento de angina estable e inestable, e infarto de miocardio $(\mathrm{IM})^{7,8}$.

El acceso femoral para la ICP fue descrito en 1967 por Judkins en tanto que el radial en 1989 por Campeau9. El abordaje femoral es considerado por muchos la técnica estándar ya que brinda control óptimo de los catéteres con escasas complicaciones trombóticas y da la posibilidad de introducir dispositivos de mayor diámetro; en los últimos años, sin embargo, ha habido un incremento en la utilización del acceso radial para la arteriografía percutánea 
coronaria tanto para los procedimientos electivos como los de emergencia en Europa y Norteamérica ${ }^{10}$. El estudio RIVAL, que incluyó 7.021 pacientes con síndrome coronario agudo a quienes se les realizó una arteriografía por abordaje radial o femoral, mostró que no hay diferencias en los desenlaces finales de muerte, IM, ECV, o muerte por IM; pero sí mostró una reducción importante en las complicaciones vasculares mayores $^{11,12}$. Los resultados de un metanálisis ${ }^{8}$ muestran que el abordaje radial se asoció con menos tasas de sangrado y de transfusiones cuando se comparó con la técnica femoral ${ }^{13}$, mejorando calidad de vida, un egreso más rápido del paciente y, por consiguiente, una reducción en los costos de hospitalización ${ }^{14}$.

Entre las limitaciones del abordaje radial se encuentran sus dificultades de aplicación en diámetros arteriales inferiores a $3 \mathrm{~mm}$ y el espasmo frecuente que se puede presentar en algunos pacientes de talla baja, lo que dificulta el avance de dispositivos. Sin embargo, el desarrollo de materiales hidrofílicos y el uso de medicación vasodilatadora hacen que cada vez sean menos frecuentes estas complicaciones. En distintos centros del mundo, el abordaje radial se emplea en menos del $10 \%$ de todos los procedimientos, evidenciando una resistencia al cambio o poco convencimiento de la técnica por parte de los cardiólogos intervencionistas ${ }^{15}$.

El propósito de este estudio fue realizar una evaluación económica de tipo costo-efectividad para comparar los beneficios clínicos y económicos del uso del acceso arterial radial comparado con el femoral en la realización de la arteriografía coronaria diagnóstica y en ICP en pacientes adultos con posible enfermedad cardiovascular, en Colombia.

\section{Materiales y métodos}

Se desarrollaron dos análisis de costo-efectividad, uno para comparar los abordajes vasculares (femoral y radial) en procedimiento diagnóstico y otro en intervención, es decir, en angioplastia coronaria transluminal percutánea. Se empleó un horizonte temporal de 1 mes, por lo que no se aplicó ninguna tasa de descuento. Este periodo se relaciona con la frecuencia de complicaciones posteriores a la realización del procedimiento reportadas en diferentes investigaciones. La perspectiva utilizada para la estimación de los costos fue la del tercero pagador (sistema de salud colombiano) por lo que se incluyeron solo costos médicos directos asociados a la ejecución de los procedimientos, sin tener en cuenta cambios en la productividad, ni gastos de bolsillo del paciente o de los cuidadores.

\section{Efectividad y seguridad}

Los árboles de decisión se diseñaron en TreeAge 2009 y comprenden los dos tipos de abordaje y las posibles complicaciones evitadas, como medida de efectividad (fig. 1).

La falla o fracaso del procedimiento se define como la necesidad de punción en un segundo acceso, es decir el requerimiento del abordaje contrario. Si no se requiere un segundo acceso el procedimiento se considera exitoso y este puede desarrollarse sin complicaciones o presentar las siguientes: eventos adversos cardiovasculares mayores que incluyen muerte, IM, cirugía de revascularización coronaria y accidente cerebrovascular; sangrado mayor; complicaciones menores (hematomas principalmente) y trombosis arterial. La frecuencia de complicaciones fue extraída de metanálisis y estudios clínicos aleatorizados. La tabla 1 agrupa las frecuencias de complicaciones evaluadas en cada uno de los abordajes para el caso base.

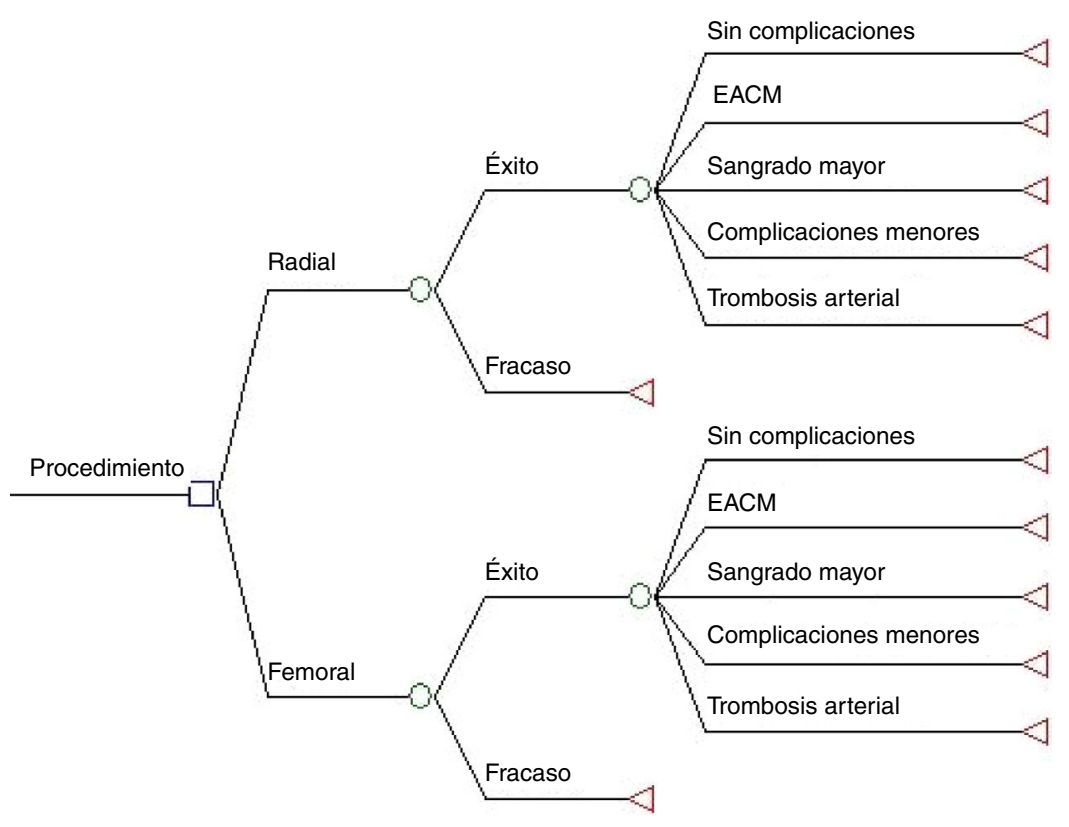

Figura 1 Modelo de decisión para el análisis de costo-efectividad del tipo de abordaje en procedimientos diagnósticos e intervencionistas

Fuente: Elaborada por los autores. 
Tabla 1 Frecuencia de complicaciones de acuerdo con el tipo de abordaje vascular

\begin{tabular}{|c|c|c|c|}
\hline Complicaciones & Radial & Femoral & Fuente \\
\hline Fallas en el procedimiento & $7,27 \%$ & $2,40 \%$ & Agostoni, $2004^{12}$ \\
\hline $\mathrm{EACM}^{\dagger}$ & & & Agostoni, $2004^{12}$ \\
\hline Muerte & $0,42 \%$ & $0,58 \%$ & Access, $1997^{16}$ Brafe, \\
\hline Infarto de miocardio & $1,08 \%$ & $1,54 \%$ & $1997^{17}$ Cooper, $1999^{18}$ \\
\hline Cirugía revascularización coronaria & $0,24 \%$ & $0,39 \%$ & Tempura, $2003^{19}$ \\
\hline Accidente cerebrovascular & $0,00 \%$ & $0,13 \%$ & Octoplus, $2004^{20}$ \\
\hline Sangrados mayores & $0,96 \%$ & $2,64 \%$ & Bertrand, $2012^{13}$ \\
\hline Complicaciones menores & $5,70 \%$ & $2,22 \%$ & Mitchell, $2012^{21}$ \\
\hline Trombosis arterial & $5,00 \%$ & $<1,00 \%$ & Agostoni, $2004^{12}$ Butman, $2010^{22}$ \\
\hline
\end{tabular}

EACM: eventos adversos cardiovasculares mayores.

\section{Costos}

En el modelo se incluyeron los costos médicos directos relacionados con la intervención y sus complicaciones aplicando la metodología de microcosteo. Los costos por evento resultantes fueron expresados en pesos colombianos del año 2016 (1 USD = COP 3.051)

La identificación y medición de recursos se realizó con base en los casos seleccionados de los pacientes a quienes se les realizó esta clase de procedimientos en el servicio de hemodinámica del hospital San Vicente Fundación, en Medellín. Se recolectó información de los recursos utilizados por cada paciente desde su ingreso a la sala de procedimientos de hemodinamia hasta su salida del servicio o cierre de la interconsulta. Se consideraron insumos, laboratorios, hospitalización, seguimiento, medicamentos y los procedimientos requeridos para el abordaje radial y femoral, tanto en diagnóstico como en intervención. Los costos de los laboratorios, hospitalización (recuperación, hemodinamia y cuidados coronarios) y procedimientos fueron estimados con base en el Manual tarifario ISS 2001 cuyas tarifas fueron ajustadas al 25\% para el mínimo, 30\% para el caso base y $48 \%$ para el máximo, de acuerdo con las recomendaciones establecidas por el IETS ${ }^{23}$. Los costos de insumos y dispositivos médicos para la realización de los diferentes procedimientos se obtuvieron de la IPS con base en la información de facturación del año 2014 por lo que se realizó el ajuste por IPC. Dentro de los insumos para arteriografía coronaria (ambos abordajes) se tuvieron en cuenta los siguientes: catéter venoso, equipos de venoclisis, equipo bomba de infusión, extensión, jeringas desechables, manifold de tres vías, guía diagnóstica. Para el abordaje radial se consideró además kit transradial, catéter coronario Jacky y pulsera de compresión radial y para el abordaje femoral aguja angiográfica, catéter diagnóstico coronario JL y JR, y hoja de bisturí. Como insumos para la angioplastia coronaria transluminal percutánea se consideraron los enunciados anteriormente más los siguientes: catéter guía, guía coronaria, balón de angioplastia y stent medicado.

Para los medicamentos se consultó el reporte de Sismed correspondiente al año 2016 y la estimación de costos se realizó mediante una búsqueda de los precios de medicamentos en el Sismed de 2016 para canal institucional y entidad laboratorio. El precio base y los valores mínimo y máximo para el análisis de sensibilidad se estableció como el promedio ponderado por unidades vendidas. Para los medicamentos y dispositivos médicos regulados (como el stent coronario medicado) se tomaron los precios reportados en las circulares emitidas por la Comisión Nacional de Precios de Medicamentos y Dispositivos Médicos (CNPMDM). En la tabla 2 se presentan los costos totales de los procedimientos diagnóstico e intervencionista para cada uno de los abordajes. En la tabla 3, se presentan los costos relacionados con fallas en los procedimientos y posibles complicaciones.

Tabla 2 Costos totales de arteriografía coronaria y angioplastia coronaria transluminal percutánea (ACTP) por tipo de acceso vascular

\begin{tabular}{|c|c|c|c|c|}
\hline \multirow[t]{2}{*}{ Tipo } & \multicolumn{2}{|c|}{ Arteriografía coronaria } & \multicolumn{2}{|c|}{ ACTP } \\
\hline & Radial & Femoral & Radial & Femoral \\
\hline Insumos & $\$ 383.797$ & $\$ 234.852$ & $\$ 383.797$ & $\$ 305.308$ \\
\hline Insumos intervención (incluye stent) & & & $\$ 5.010 .207$ & $\$ 5.452 .673$ \\
\hline Laboratorio & $\$ 113.815$ & $\$ 62.803$ & $\$ 113.815$ & $\$ 45.078$ \\
\hline Hospitalización & $\$ 100.766$ & $\$ 411.219$ & $\$ 411.219$ & $\$ 411.219$ \\
\hline Seguimiento & $\$ 19.624$ & $\$ 19.624$ & $\$ 19.624$ & $\$ 19.624$ \\
\hline Medicamentos & $\$ 307.205$ & $\$ 289.735$ & $\$ 925.199$ & $\$ 917.729$ \\
\hline Procedimiento & $\$ 450.808$ & $\$ 450.808$ & $\$ 1.161 .524$ & $\$ 1.161 .524$ \\
\hline Total & $\$ 1.376 .015$ & $\$ 1.469 .041$ & $\$ 8.025 .386$ & $\$ 8.313 .154$ \\
\hline
\end{tabular}


Tabla 3 Costo de fallas y complicaciones relacionadas con los procedimientos

\begin{tabular}{|c|c|c|c|c|c|}
\hline & Complicaciones & Min & Caso base & $\operatorname{Max}$ & Fuente \\
\hline \multicolumn{2}{|c|}{ Fallas en el procedimiento diagnóstico con abordaje radial } & $\$ 1.228 .348$ & $\$ 1.284 .968$ & $\$ 1.422 .003$ & Microcosteo \\
\hline \multicolumn{2}{|c|}{ Fallas en el procedimiento diagnóstico con abordaje femoral } & $\$ 1.932 .399$ & $\$ 2.011 .623$ & $\$ 2.179 .843$ & Microcosteo \\
\hline \multicolumn{2}{|c|}{ Fallas en el procedimiento intervencionista con abordaje radial } & $\$ 1.861 .758$ & $\$ 1.933 .133$ & $\$ 2.093 .504$ & Microcosteo \\
\hline \multicolumn{2}{|c|}{ Fallas en el procedimiento intervencionista con abordaje femoral } & $\$ 1.932 .399$ & $\$ 2.011 .623$ & $\$ 2.179 .843$ & Microcosteo \\
\hline \multirow[t]{4}{*}{ EACM } & Muerte & $\$ 1.193 .957$ & $\$ 1.371 .765$ & $\$ 1.740 .113$ & \\
\hline & Infarto de miocardio & $\$ 7.106 .979$ & $\$ 9.863 .323$ & $\$ 48.248 .643$ & Castellanos, $2011^{4}$ \\
\hline & Cirugía revascularización coronaria & $\$ 2.236 .988$ & $\$ 2.326 .467$ & $\$ 2.648 .593$ & ISSS, $2001^{24}$ \\
\hline & Accidente cerebrovascular & $\$ 9.750 .020$ & $\$ 10.468 .943$ & $\$ 26.144 .202$ & Castañeda, $2014^{25}$ \\
\hline \multicolumn{2}{|c|}{ Sangrados mayores } & $\$ 199.050$ & $\$ 207.012$ & $\$ 235.675$ & ISSS, $2001^{24}$ \\
\hline \multicolumn{2}{|c|}{ Complicaciones menores } & $\$ 32.010$ & $\$ 35.567$ & $\$ 39.124$ & ISSS, $2001^{24}$ \\
\hline \multicolumn{2}{|c|}{ Trombosis arterial } & $\$ 33.032$ & $\$ 36.702$ & $\$ 40.372$ & ISSS, $2001^{24}$ \\
\hline
\end{tabular}

\section{Resultados}

\section{Arteriografía coronaria}

El costo promedio de una arteriografía coronaria por acceso radial es de $\$ 1.384 .945$ y de $\$ 1.474 .543$ por acceso femoral. De acuerdo con los resultados de la tabla 4 el costo de una arteriografía coronaria exitosa es inferior en el acceso radial que en el femoral, con una diferencia de \$96.222; sin embargo, hay mayor proporción de fallas en el procedimiento. Para todos los casos con complicaciones, el costo por evento con el uso del acceso radial es inferior al del abordaje femoral; no obstante, se le atribuyen mayores frecuencias de complicaciones relacionadas con fallas en el procedimiento, eventos adversos menores y trombosis arterial. Al evaluar el costo incremental por complicación evitada se observa que el abordaje radial es una alternativa dominante respecto al acceso femoral en las complicaciones de mayor severidad (eventos adversos cardiovasculares mayores y sangrado mayor) ya que tiene menor costo y presenta menos complicaciones que el acceso femoral. Al hacer un análisis para todas las complicaciones el abordaje radial resulta desfavorecido frente al femoral en fallas en el procedimiento, que son dependientes del operador, o en el desarrollo de complicaciones menores y trombosis arterial, siendo esta última una complicación casi exclusiva del abordaje radial. Aunque la incidencia de fallas en el procedimiento, complicaciones menores y trombosis arterial es mayor en el acceso radial que en el femoral, el sobrecosto asociado a su presentación es poco representativo respecto al costo total del procedimiento. Puede verse que el costo incremental por complicaciones de menor severidad evitadas (fallas en el procedimiento, menores, trombosis arterial) para el abordaje femoral es cercano a los $\$ 25.000$ por complicación evitada. Puede verse que a pesar de que el abordaje radial presenta más casos de complicaciones menores tiene un costo inferior por evento, con una diferencia de $\$ 89.598 .000$ por cada 1.000 procedimientos diagnósticos.

En la figura 2 se presentan los resultados del análisis de sensibilidad univariado mediante diagrama de tornado que permite observar el impacto de la modificación de las distintas variables sobre la estimación. Las variables que generan mayor incertidumbre en el modelo son los costos de hospitalización, de insumos y de laboratorios.

\section{Angioplastia coronaria transluminal percutánea}

El costo ponderado total de una angioplastia coronaria transluminal percutánea por abordaje radial es de $\$ 8.037 .743$ y de $\$ 8.319 .178$ por abordaje femoral. De acuerdo con los resultados de la tabla 5 el costo de una angioplastia coronaria transluminal percutánea exitosa por acceso radial es inferior al del acceso femoral, con

Tabla 4 Comparación de costos y complicaciones entre la arteriografía coronaria con abordaje radial vs. femoral

\begin{tabular}{|c|c|c|c|c|c|c|c|}
\hline \multirow{2}{*}{$\begin{array}{l}\text { Resultados del } \\
\text { procedimiento }\end{array}$} & \multicolumn{2}{|c|}{ (\% casos) } & \multirow{2}{*}{$\begin{array}{l}\text { Diferencias de } \\
\text { efectividad }\end{array}$} & \multicolumn{2}{|c|}{ Costos por paciente } & \multirow{2}{*}{$\begin{array}{l}\text { Costos } \\
\text { incrementales }\end{array}$} & \multirow{2}{*}{$\begin{array}{l}\text { Razón de } \\
\text { costo-efectividad } \\
\text { incremental }\end{array}$} \\
\hline & Radial & Femoral & & Radial & Femoral & & \\
\hline Exitoso & 92,7 & 97,6 & $-4,9$ & $\$ 1.378 .321$ & $\$ 1.474 .543$ & $\$ 96.222$ & $\$ 19.637$ \\
\hline Sin complicaciones & 86,6 & 91,5 & $-4,9$ & $\$ 1.376 .015$ & $\$ 1.469 .041$ & $-\$ 93.026$ & $\$ 18.985$ \\
\hline $\mathrm{MACE}^{\dagger}$ & 1,7 & 2,6 & $-0,9$ & $\$ 1.494 .931$ & $\$ 1.651 .575$ & $-\$ 156.644$ & Dominante \\
\hline Sangrado mayor & 1,0 & 2,6 & $-1,6$ & $\$ 1.377 .866$ & $\$ 1.474 .133$ & $-\$ 96.267$ & Dominante \\
\hline Complicaciones menores & 5,7 & 2,2 & 3,5 & $\$ 1.378 .042$ & $\$ 1.469 .831$ & $-\$ 91.789$ & $\$ 26.225$ \\
\hline Trombosis arterial & 5,0 & 1,0 & 4,0 & $\$ 1.377 .850$ & $\$ 1.469 .408$ & $-\$ 91.558$ & $\$ 22.890$ \\
\hline Fallas procedimiento & 7,3 & 2,4 & 4,9 & $\$ 1.469 .432$ & $\$ 1.496 .004$ & $-\$ 26.572$ & $\$ 5.423$ \\
\hline
\end{tabular}

\footnotetext{
$\dagger$ MACE: Eventos adversos cardiovasculares mayores.
} 


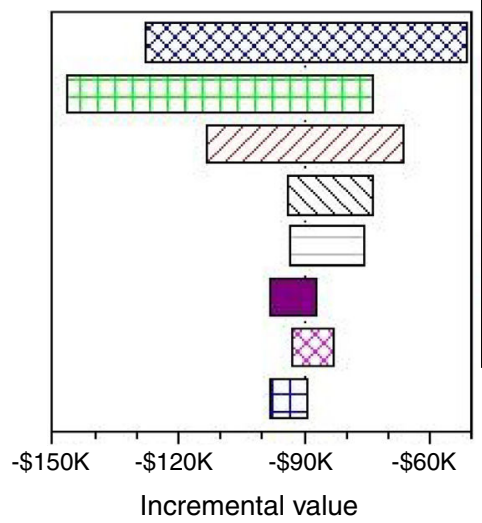

囚 c_insumos_rad: 345418 to 422177
$\square$ c_hospitalizacion_fem: 395403 to 468157
$\square$ c_insumos_fem: 211367 to 258337
$\square$ c_laboratorios_rad: 109438 to 129574
$\square$ c_hospitalizacion_rad: 96890 to 114718
c_laboratorios_fem: 60388 to 71499
c_heparina: 6500 to 16750
$\boxplus$ c_infarto: 7106979 to 48248643

Figura 2 Análisis de sensibilidad de costos: diagrama de tornado para el uso de abordaje femoral vs. radial en arteriografía coronaria.

Tabla 5 Comparación de costos y complicaciones entre angioplastia coronaria transluminal percutánea con abordaje radial vs. femoral

\begin{tabular}{|c|c|c|c|c|c|c|c|}
\hline \multirow{2}{*}{$\begin{array}{l}\text { Resultados del } \\
\text { procedimiento }\end{array}$} & \multicolumn{2}{|c|}{ (\% casos) } & \multirow{2}{*}{$\begin{array}{l}\text { Diferencias de } \\
\text { efectividad }\end{array}$} & \multicolumn{2}{|c|}{ Costos por paciente } & \multirow{2}{*}{$\begin{array}{l}\text { Costos } \\
\text { incrementales }\end{array}$} & \multirow{2}{*}{$\begin{array}{l}\text { Razón de costo- } \\
\text { efectividad } \\
\text { incremental }\end{array}$} \\
\hline & Radial & Femoral & & Radial & Femoral & & \\
\hline Exitoso & 92,7 & 97,6 & $-4,9$ & $\$ 8.027 .693$ & $\$ 8.318 .139$ & $-\$ 290.446$ & $\$ 59.275$ \\
\hline Sin complicaciones & 86,6 & 91,5 & $-4,9$ & $\$ 8.025 .386$ & $\$ 8.313 .155$ & $-\$ 287.769$ & $\$ 58.728$ \\
\hline $\mathrm{MACE}^{\dagger}$ & 1,7 & 2,6 & $-0,9$ & $\$ 8.144 .302$ & $\$ 8.495 .689$ & $-\$ 351.387$ & Dominante \\
\hline Sangrado mayor & 1,0 & 2,6 & $-1,6$ & $\$ 8.027 .373$ & $\$ 8.318 .620$ & $-\$ 291.247$ & Dominante \\
\hline Complicaciones menores & 5,7 & 2,2 & 3,5 & $\$ 8.027 .413$ & $\$ 8.313 .945$ & $-\$ 286.532$ & $\$ 81.866$ \\
\hline Trombosis arterial & 5,0 & 1,0 & 4,0 & $\$ 8.027 .221$ & $\$ 8.313 .522$ & $-\$ 286.301$ & $\$ 71.575$ \\
\hline Fallas procedimiento & 7,3 & 2,4 & 4,9 & $\$ 8.165 .925$ & $\$ 8.361 .434$ & $-\$ 195.509$ & $\$ 39.900$ \\
\hline
\end{tabular}

$\dagger$ MACE: eventos adversos cardiovasculares mayores.

una diferencia de $\$ 290.446$. En angioplastia coronaria transluminal percutánea, al evaluar el costo incremental por complicación evitada, se observa que el abordaje radial es una alternativa dominante respecto al acceso femoral en las complicaciones de mayor severidad (eventos adversos cardiovasculares mayores y sangrado mayor). Similar a los resultados obtenidos con el proceso diagnóstico, al agrupar todas las complicaciones el abordaje radial resulta menos favorecido, por la diferencia en complicaciones como fallas en el procedimiento, complicaciones menores y trombosis arterial. Para este caso el costo incremental por complicaciones evitadas de menor severidad (fallas en el procedimiento, menores, trombosis arterial) para el abordaje femoral oscila entre los \$300.000$\$ 400.000$. El abordaje radial puede llegar a representar un ahorro de $\$ 290.446 .000$ por cada 1000 intervenciones.

En la figura 3 se presentan los resultados del análisis de sensibilidad univariado. En términos de costos, las variables

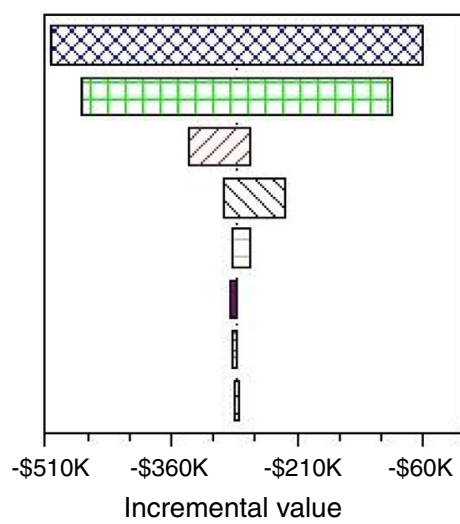

囚C_insumos_fem: 5536217 to 5979745

$\square$ c_insumos_rad: 5208638 to 5579371

乙C_hospitalizacion_fem: 395403 to 468157

$\triangle$ c_hospitalizacion_rad: 395403 to 468157

曰c_laboratorios_rad: 109438 to 129574

c_infarto: 7106979 to 48248643

QC_laboratorios_fem: 45344 to 51319

$\boxplus$ C_nitroglicerina: 3900 to 8000

Figura 3 Análisis de sensibilidad de costos: diagrama de tornado para el uso de abordaje radial en angioplastia coronaria transluminal percutánea (ACTP). 
que generan mayor incertidumbre son los costos de los insumos y de hospitalización.

\section{Discusión}

De acuerdo con estos resultados, el abordaje radial presenta una reducción anual de los eventos adversos cardiovasculares mayores y sangrado mayor, y un aumento en el número de complicaciones relacionadas con fallas en el procedimiento, complicaciones menores y de trombosis arterial. El abordaje radial tanto en arteriografía diagnóstica como en angioplastia coronaria transluminal percutánea es una alternativa dominante para los eventos adversos mayores, pues presenta menores costos y menores complicaciones que el abordaje femoral. De acuerdo con el análisis de sensibilidad univariado los costos de hospitalización y de insumos requeridos en los procedimientos son los que generan mayor incertidumbre en el modelo.

La influencia limitada de las complicaciones en el incremento de los costos de los procedimientos se relaciona con la baja frecuencia de presentación, de acuerdo con lo reportado en la literatura. Por otro lado, las complicaciones relacionadas con eventos adversos cardiovasculares mayores, que son las que generan mayor costo en la atención, son menores con el acceso radial por lo que constituye un factor determinante en la selección de un abordaje particular. La reducción del sangrado mayor favorece al acceso radial en términos clínicos, y tiene alto impacto en el ahorro en la medida que se incrementa el número de procedimientos. Las fallas en el procedimiento, complicaciones menores y la trombosis arterial hacen que el abordaje sea menos ahorrador respecto al caso base, por lo que la destreza del operador y una selección y preparación adecuada de los pacientes, constituyen factores críticos en la selección del tipo de acceso.

De acuerdo con los resultados obtenidos, el abordaje radial podría representar un ahorro respecto al abordaje femoral cercano a 89 millones anuales en el cateterismo cardiaco diagnóstico, y de $\$ 290$ millones anuales en angioplastia coronaria transluminal percutánea por cada 1.000 procedimientos realizados.

Los resultados de esta investigación concuerdan con algunas investigaciones disponibles en la literatura. Roussanov et al. ${ }^{26}$ evaluaron la costo-efectividad del acceso radial en comparación con el femoral en cateterización cardiaca diagnóstica. El costo total del procedimiento incluyendo equipos e insumos y estancia en sala de recuperación fue significativamente menor en el abordaje radial que en el grupo femoral (369,5 USD \pm 74,6 USD vs. 446,9 USD \pm 60,2 USD $p<0,001)$. Los autores señalan que aunque el ahorro por caso puede parecer pequeño, con la cantidad de cateterismos cardiacos realizados anualmente existe un potencial de ahorro con el abordaje radial ${ }^{26}$.

Mitchelli et $\mathrm{al}^{21}$ llevaron a cabo un análisis de costobeneficio del acceso arterial radial para angiografía coronaria e intervención. En un metaanálisis encontraron que la cateterización radial incrementó significativamente las fallas relacionadas con la cateterización (OR 4,92; IC 95\% 2,69-8,98) pero redujo las complicaciones mayores (OR 0,32 ; IC $95 \%$ 0,24-0,42) y el sangrado mayor (OR 0,39; IC $95 \%$ $0,27-0,57)$ en comparación con el acceso femoral. No hubo diferencias en las tasas de éxito de los procedimientos o los eventos adversos cardiovasculares mayores. Encontraron que el abordaje radial costó 275 USD (IC95\% - 374 USD a -183 USD) menos por paciente que el acceso femoral, desde la perspectiva institucional ${ }^{21}$.

En la guía NICE de infarto agudo de miocardio con elevación del segmento ST (STEMI) se evaluó el beneficio clínico y la costo-efectividad del acceso radial comparado con el femoral para angiografía coronaria y en seguimiento de intervención coronaria percutánea primaria en pacientes con STEMI. El análisis mostró que los costos de los equipos para procedimiento femoral estándar son ligeramente más altos que los de los procedimientos radiales. La diferencia potencial final en costos se debió a una reducción de la estancia en pacientes con acceso radial. El análisis mostró que es probable que el abordaje radial en intervención coronaria percutánea primaria sea menos costoso y la diferencia puede variar entre 50 GBP y 150 GBP. En el análisis de costo-efectividad el acceso radial resultó dominar al acceso femoral respecto a la mortalidad por todas las causas a corto plazo, sangrado mayor y sangrado menor. Sin embargo, los resultados dependen en gran medida de la generalización que se pueda hacer de los resultados de los estudios clínicos ${ }^{27}$.

La principal limitación de este estudio se relaciona con que la frecuencia de complicaciones proviene de estudios internacionales, y no se dispone de información local. En cuanto a dicho punto, no se tuvieron en cuenta complicaciones diferenciales entre los procesos diagnósticos e intervencionistas. En el modelo se consideró como uno de los supuestos que la experticia del operador responsable del procedimiento es similar en el abordaje radial $y$ en el femoral por lo que no afecta los resultados; sin embargo, en el contexto real las tasas de éxito son dependientes del operador de ahí que esta variable será particularmente importante en el contexto de hospitales o clínicas de enseñanza y esto refuerza la necesidad de generación de datos multicéntricos acerca de las frecuencia de éxito y falla en este tipo de procedimientos. La validez externa del modelo se relaciona con los supuestos establecidos y el horizonte temporal definido.

\section{Conclusiones}

El abordaje radial en arteriografía coronaria diagnóstica y en angioplastia coronaria transluminal percutánea mostró ser dominante respecto al abordaje femoral para los eventos adversos cardiovasculares mayores, ya que se relaciona con una menor frecuencia de complicaciones mayores y presenta un menor costo.

\section{Conflicto de intereses}

Ninguno.

\section{Bibliografía}

1. Randomised trial of intravenous streptokinase, oral aspirin, both, or neither among 17,187 cases of suspected acute myocardial infarction: ISIS-2. ISIS-2 (Second International Study of Infarct Survival) Collaborative Group. Lancet. 1988;2:349-60. 
2. Rosselli D, Tarazona N, Aroca A. La salud en Colombia 1953-2013: Un análisis de estadísticas vitales. Medicina. 2014;36:120-35.

3. Moher D, Liberati A, Tetzlaff J, Altman DG. Preferred reporting items for systematic reviews and meta-analyses: the PRISMA statement. Ann Intern Med. 2009;151:264-9. W64.

4. Gómez JE. Morbimortalidad cardiovascular en el mundo. Rev Colomb Cardiol. 2012;19:298-9.

5. Instituto Nacional de Salud. Las enfermedades cardiovasculares: un problema de salud pública y un reto global. Biomédica. 2011;31:469-73.

6. Estornell Erill J. Non-invasive coronary angiography with multislice CT: at last an alternative to conventional coronary angiography? Rev Esp Cardiol. 2004;57:198-200.

7. Members C, Gibbons RJ, Abrams J, Chatterjee K, Daley J, Deedwania PC, et al. ACC/AHA 2002 Guideline update for the management of patients with chronic stable angina. Circulation. 2003;107:149-58.

8. Anderson JL, Adams CD, Antman EM, Bridges CR, Califf RM, Casey DE, et al. 2011 ACCF/AHA Focused update incorporated into the ACC/AHA 2007 Guidelines for the management of patients with unstable angina/non-ST-elevation myocardial infarction. Circulation. 2011;123:e426-579.

9. Bucciarelli-Ducci C, Pennell DJ. Low diagnostic yield of elective coronary angiography. N Engl J Med. 2010;363:94.

10. Mamas MA, Ratib K, Routledge H, Neyses L, Fraser DG, de Belder $M$, et al. Influence of arterial access site selection on outcomes in primary percutaneous coronary intervention: are the results of randomized trials achievable in clinical practice? JACC Cardiovasc Interv. 2013;6:698-706.

11. Sanmartín M, Goicolea J, Meneses D, Ruiz-Salmerón R, Mantilla $\mathrm{R}$, Claro R, et al. Coronary angiography with $4 \mathrm{~F}$ catheters by the radial: minimally invasive catheterization. Rev Esp Cardiol. 2003; 56:145-51.

12. Agostoni P, Biondi-Zoccai GGL, de Benedictis ML, Rigattieri S, Turri M, Anselmi M, et al. Radial versus femoral approach for percutaneous coronary diagnostic and interventional procedures; Systematic overview and meta-analysis of randomized trials. J Am Coll Cardiol. 2004;44:349-56.

13. Bertrand OF, Bélisle P, Joyal D, Costerousse O, Rao SV, Jolly SS, et al. Comparison of transradial and femoral approaches for percutaneous coronary interventions: a systematic review and hierarchical Bayesian meta-analysis. Am Heart J. 2012;163:632-48.

14. Pandie S, Mehta SR, Cantor WJ, Cheema AN, Gao P, Madan $M$, et al. Radial versus femoral access for coronary angiography/intervention in women with acute coronary syndromes: insights from the RIVAL Trial (Radial vs femoral access for coronary intervention). JACC Cardiovasc Interv. 2015;8:505-12.

15. Mehta SR, Boden WE, Eikelboom JW, Flather M, Steg PG, Avezum A, et al. Antithrombotic therapy with fondaparinux in relation to interventional management strategy in patients with ST- and non-ST-segment elevation acute coronary syndromes: an individual patient-level combined analysis of the Fifth and Sixth Organization to Assess Strategies in Ischemic Syndromes
(OASIS 5 and 6) randomized trials. Circulation. 2008;118: 2038-46.

16. Kiemeneij F, Laarman GJ, Odekerken D, Slagboom T, van der Wieken R. A randomized comparison of percutaneous transluminal coronary angioplasty by the radial, brachial and femoral approaches: the access study. J Am Coll Cardiol. 1997;29:1269-75.

17. Benit E, Missault L, Eeman T, Carlier M, Muyldermans L, Materne P, et al. Brachial, radial, or femoral approach for elective Palmaz-Schatz stent implantation: a randomized comparison. Cathet Cardiovasc Diagn. 1997;41:124-30.

18. Cooper CJ, El-Shiekh RA, Cohen DJ, Blaesing L, Burket MW, Basu $A$, et al. Effect of transradial access on quality of life and cost of cardiac catheterization: A randomized comparison. Am Heart J. 1999;138 3 Pt 1:430-6.

19. Saito S, Tanaka S, Hiroe Y, Miyashita Y, Takahashi S, Tanaka $\mathrm{K}$, et al. Comparative study on transradial approach vs. transfemoral approach in primary stent implantation for patients with acute myocardial infarction: results of the test for myocardial infarction by prospective unicenter randomization for access sites (TEMPURA) trial. Catheter Cardiovasc Interv. 2003;59:26-33.

20. Louvard Y, Benamer H, Garot P, Hildick-Smith D, Loubeyre C, Rigattieri S, et al. Comparison of transradial and transfemoral approaches for coronary angiography and angioplasty in octogenarians (the OCTOPLUS study). Am J Cardiol. 2004;94:1177-80.

21. Mitchell MD, Hong JA, Lee BY, Umscheid CA, Bartsch SM, Don CW. Systematic review and cost-benefit analysis of radial artery access for coronary angiography and intervention. Circ Cardiovasc Qual Outcomes. 2012;5:454-62.

22. Butman SM. Complications of percutaneous coronary interventions. New York, NY: Springer Science \& Business Media; 2010.

23. Díaz M, Peña E, Mejía A. Manual metodológico para la elaboración de evaluaciones de efectividad seguridad y validez diagnóstica de tecnologías en salud. Bogotá D.C: Instituto de Evaluación Tecnológica en Salud - IETS; 2014.

24. Instituto de Seguros Sociales. Acuerdo 256 de 2001. Manual de tarifas de la Entidad Promotora de Salud EPS-ISS [Internet]. 2001. Recuperado a partir de: https: / / lexsaludcolombia.files.wordpress.com/2010/10/tarifasiss-2001.pdf.

25. Castañeda-Cardona C, Coral-Casas J, Rueda JD, Díaz CE, Rueda MC, Rosselli D. Análisis de costos de atención de infarto cerebral agudo con o sin fibrilación auricular. Acta Neurol Colomb. 2014;30:78-82.

26. Roussanov O, Wilson SJ, Henley K, Estacio G, Hill J, Dogan B, et al. Cost-effectiveness of the radial versus femoral artery approach to diagnostic cardiac catheterization. J Invasive Cardiol. 2007;19:349-53.

27. National Clinical Guideline Centre. Radial versus femoral arterial access for primary percutaneous coronary intervention. En: NICE Clinical Guidelines, No 167 [Internet]. 2013:114-29. Disponible en: https://www.ncbi.nlm.nih.gov/books/NBK259079/. [consultado 2 Ago 2017]. 